\title{
THE STELLAR SPHEROID, THE DISK, AND THE DYNAMICS OF THE COSMIC WEB
}

\author{
R. Domínguez-Tenreiro ${ }^{1}$, A. Obreja ${ }^{1}$, C. B. Brook ${ }^{1}$, F. J. Martínez-Serrano ${ }^{2,4}$, G. Stinson ${ }^{3}$, And A. Serna ${ }^{2}$ \\ ${ }^{1}$ Dept. de Física Téórica, Univ. Autónoma de Madrid, E-28049 Cantoblanco Madrid, Spain; rosa.dominguez@uam.es \\ ${ }^{2}$ Dept. de Física y A.C., Universidad Miguel Hernández, E-03202 Elche, Spain \\ ${ }^{3}$ Max-Planck-Institut für Astronomie, Königstuhl 17, D-69117, Heidelberg, Germany \\ ${ }^{4}$ Next Limit Dynamics SL, Angel Cavero 2, E-28048 Madrid, Spain \\ Received 2014 November 5; accepted 2015 January 2; published 2015 February 20
}

\begin{abstract}
Models of the advanced stages of gravitational instability predict that baryons that form the stellar populations of current galaxies at $z=0$ displayed a web-like structure at high $z$, as part of the cosmic web (CW). We explore details of these predictions using cosmological hydrodynamical simulations. When the stellar populations of the spheroid and disk components of simulated late-type galaxies are traced back separately to high $z \mathrm{~s}$ we found CWlike structures where spheroid progenitors are more evolved than disk progenitors. The distinction between the corresponding stellar populations, as driven by their specific angular momentum content $j$, can be explained in terms of the CW evolution, extended to two processes occurring at lower $z$. First, the spheroid progenitors strongly lose $j$ at collapse, which contrasts with the insignificant $j$ loss of the disk progenitors. The second is related to the lack of alignment, at assembly, between the spheroid-to-be material and the already settled proto-disk, in contrast to the alignment of disk-to-be material, in some cases resulting from circumgalactic, disk-induced gravitational torques. The different final outcomes of these low- $z$ processes have their origins in the different initial conditions driven by the $\mathrm{CW}$ dynamics.
\end{abstract}

Key words: cosmology: theory - galaxies: formation - methods: numerical

\section{INTRODUCTION}

The idea that galaxies get most of their gas through cold, dense streams (i.e., the so-called "cold mode" for galaxy mass assembly) was first formulated by Binney (1977) and (re) discovered in the last decade (Birnboim \& Dekel 2003; Kereš et al. 2005; Ocvirk et al. 2008; Brooks et al. 2009; van de Voort et al. 2011). This scenario has received a lot of attention in the last few years because it helps to explain DM halo and galaxy angular momentum acquisition through filaments (see Kimm et al. 2011; Pichon et al. 2011; Codis et al. 2012; Danovich et al. 2012, 2014; Tillson et al. 2012; Stewart et al. 2013; Dubois et al. 2014; Welker et al. 2014), and, in addition, for its possible implications in the star formation history of galaxies (Brooks et al. 2009; Kereš et al. 2009; Obreja et al. 2013).

In the context of the theories for the advanced stages of gravitational instability, cold streams feeding clumps is a general prediction of the Zel'dovich approximation (Zel'dovich 1970) extended to the Adhesion Model (see e.g., Gurbatov et al. 1989; Kofman et al. 1990; Gurbatov et al. 2012), predictions now confirmed in detail by numerical simulations (Cautun et al. 2014). Indeed, the Adhesion Model predicts that, at a given scale, walls surrounding voids, filaments and nodes (i.e., the Cosmic Web, CW, elements, see Bond et al. 1996) successively emerge in multi-streaming regions. Then they vanish as mass elements flow through the voids toward the walls, through the walls toward the filaments, and finally from filaments to nodes, where mass elements pill up and virialize after the web-like mass configuration, at the scale considered, turns around and collapses (see, e.g., Domínguez-Tenreiro 2011). Meanwhile, the same CW elements emerge at larger and larger scales, disappearing later on in favor of voids and nodes.

These considerations strongly suggest that the stellar populations of current galaxies displayed a web-like structure as part of the $\mathrm{CW}$ at high $z$, later on removed due to the $\mathrm{CW}$ evolution. The question arises if the $\mathrm{CW}$ dynamics might also explain the origin of the fine structure of late-type galaxies (hereafter, LTGs). Indeed, stellar populations of LTGs come in three different categories, those forming the spheroid (i.e., the bulge and stellar halo), the thick disk, and the thin disk (see Mould 1982; Gilmore et al. 1989; Ivezić et al. 2012 for reviews), whose different properties are thought to be driven by their different specific angular momentum $j$ content. We see that its acquisition by galaxies is a key piece of the debate.

For a typical disk galaxy the total $j^{5}$ enclosed within the Lagrangian patch associated with its halo, $j_{\text {patch }}$, grows as predicted by the Tidal Torque Theory (based on the Zel'dovich approximation; see Peebles 1969; Doroshkevich 1970; White 1984) prior to patch maximum expansion. Later on, $j_{\text {patch }}$ is roughly conserved, but redistributed (Pichon et al. 2011). Indeed, as filaments form from the collapse of matter flows coming from opposite directions, the flows coherently wind up (Codis et al. 2012) into whirls with their spin $s$ directions roughly aligned with the filament. At the same time, matter elements also acquire a net transverse motion (i.e., an orbital $j$ relative to the patch center), due to the asymmetric forces acting on filaments from the voids they divide.

Once the filaments form, they collimate longitudinal motions toward the growing central mass accumulation, coherently transporting $j$ either as clumpy or diffuse material. Pichon et al. (2011) show that, at a given time, the $j$ content of infalling matter grows with its distance to the patch center (lever effect). The implications this ordering has on $j$ acquisition by gas at the scales of galaxy halos has been analyzed by Sales et al. (2012) for Milky Way sized galaxies, and by Stewart et al. (2013) who pointed out the importance of fresh gaseous accretion to increase the $j$ content in the inner halo as time progresses (a reformulation of the lever effect previously described). Recently Danovich et al. (2014) have confirmed these ideas,

\footnotetext{
Relative to the patch center where the galaxy is to be assembled.
} 
and have shown that gravitational torques from the early settled disk cause gas and, to a lesser extent, star alignments at the circumgalactic region.

We see that new ideas have recently emerged to explain $j$ acquisition by gas. One of the still unaswered questions is why do some late type galaxies have a spheroid at all. More specifically, did these spheroid stellar populations lose most of the $j$ they have acquired at high $z$, as discussed above, or they have always been $j$-poor? What are the differences between the progenitors of spheroid and disk stellar populations? The specific purpose of this Letter is to contribute to answer these questions in the context of $\mathrm{CW}$ dynamics.

\section{METHODS AND RESULTS}

Hydrodynamical simulations in a cosmological context are a helpful tool to address the above questions. For a proper spheroid and disk characterization, one needs to measure precise differences in morphology, kinematics, and composition. Therefore, accurate conservation of angular momentum and a detailed implementation of chemical evolution are two fundamental issues. Moreover, we also need to consider the effects of discrete energy injection by stellar physics. Comparisons among the results of different codes are advisable, because we are looking for effects coming from a generic and fundamental level of physical description.

In view of these considerations, we present results of simulations run with two different SPH codes. P-DEVA (Serna et al. 2003; Martínez-Serrano et al. 2008) is an entropyconserving AP3M code where the main concern in its design was $j$ conservation. Chemical evolution makes use of the $Q_{i j}$ formalism (Talbot \& Arnett 1973). Stellar feedback is implicitly implemented through (inefficient) SF parameters, as discussed in Agertz et al. (2011). We also use the code GASOLINE (Wadsley et al. 2004), in which supernovae feedback is implemented using the blastwave formalism (Stinson et al. 2006, 2013).

To run the simulations, a zoom-in technique has been used with baryon particle masses of $m_{B}=3.79-3.74 \times 10^{5} M_{\odot}$ and initial gaseous mass of $m_{\text {gas }}=0.25-2.0 \times 10^{5} M_{\odot}$, and gravitational softening of $\epsilon_{g}=400 \mathrm{~h}^{-1} \mathrm{pc}^{-1}$ and $\epsilon_{g}=315.5-$ $156.2 \mathrm{pc}$ in P-DEVA and GASOLINE, respectively. We base our analysis on a P-DEVA set of four galaxies (LD-5101A, LD-5003A, HD-5103B, and HD-5004A) and a GASOLINE set of two galaxies (g1536- $L^{*}$ and g5664-MW), with $z=0$ stellar masses in the ranges of $M_{*}=1.29-3.26$ (P-DEVA set) and 2.32-2.50 $\times 10^{10} M_{\odot}$ (GASOLINE set). The total numbers of stellar particles (including the stellar halo) are in the range $N_{\text {star }}=33990-83101$ (P-DEVA set), and 582684 and 633218 for the two GASOLINE galaxies. P-DEVA galaxies have been studied by Doménech-Moral et al. (2012) at $z=0$, who analyze some aspects of their fine structure. Galaxy g1536- $L^{*}$ has been studied by Brook et al. (2012b) and Obreja et al. (2014). In addition, Obreja et al. (2013) who addressed bulge formation, and Domínguez-Tenreiro et al. (2014), who analyzed their $\mathrm{HI}$ and $\mathrm{H} 2$ content, SEDs, and colors, included galaxies run using both codes. In all these cases, the consistency with observational data is very satisfactory, including their bulge-to-disk luminosity ratios (see Table 1 in Domínguez-Tenreiro et al. 2014 for those in the $r$ band).

The $k k$-means method has been used by Doménech-Moral et al. (2012) to disentangle the fine structure of simulated
LTGs. This is an unsupervised clustering algorithm (Schölkopf et al. 1998; Dhillon et al. 2004; Karatzoglou et al. 2004) looking for clusters in a three-dimensional space of LTG stellar particle kinematic variables. Specifically, for each stellar particle, $i$, in a given galaxy, its total binding energy, $E_{i}$, $\epsilon_{J, i} \equiv j_{z, i} / J_{C, i}\left(E_{i}\right)$, and $j_{p, i} / J_{C, i}\left(E_{i}\right)$ have been used as variables, where $j_{z, i}\left(j_{p, i}\right)$ are the particle angular momentum projections on the disk axis (plane) and $J_{C, i}\left(E_{i}\right)$ is the angular momentum of a circular orbit with energy $E_{i}$. By applying this method, with no priors assumed apart from the number of clusters to look for, the fine structure of galaxies emerges naturally (see Figure 7 in Doménech-Moral et al. 2012, where more details can be found). Once classified, the characteristics of spheroid, thick disk, and thin disk star observational properties are recovered, as well as their mutual correlations (Doménech-Moral et al. 2012; Obreja et al. 2013; A. Obreja et al. 2015, in preparation). The same is true for GASOLINE galaxies, although using a different classification scheme (Brook et al. 2012a).

To decipher the imprints left by the CW dynamics onto current stellar populations, we have traced back these populations to their progenitors at high $z$. Specifically, for each simulated galaxy we have identified all the stellar particles belonging to each component at $z=0$ and traced these particles back to different high redshifts $z_{\text {high }}$. By plotting the positions of all the progenitors together, we confirm that indeed they display a clear CW-like structure before collapse, whose evolution follows the Adhesion Model. A main filament can be identified in this structure (Danovich et al. 2014; Dubois et al. 2014), with the disk initially normal to this filament (Figure 1, lower panel), as expected. Moreover, the systematics of $j$ acquisition by disks at the halo scale is recovered (see Section 1).

If we separately plot the positions of the progenitors of each component, we still find $\mathrm{CW}$-like configurations, but with different characteristics. In Figure 1 we plot, for a galaxy in the P-DEVA set, the projections on a given plane of the progenitor positions at $z_{\text {high }}=3.5$ and 1.45 (roughly at proto-object collapse). The left panel corresponds to the (thin) disk progenitors $^{6}$, while the right panel corresponds to the spheroid progenitors. The visual impression is that spheroid progenitors have been involved in multistreaming events from earlier on than disk progenitors, in such a way that at a given $z$, they form a CW-like structure whose dynamical state is more advanced than the $\mathrm{CW}$ corresponding to the thin disk. This more advanced stage of gravitational instability can be characterized mainly through the distributions of density and vorticity of gas elements, which are biased toward higher values than less advanced ones; see below. In addition, it implies (1), the tendency for spheroid-to-be particles to be closer to the system center of mass (c.o.m.) than disk progenitors, and (2), more stars formed within the (disconnected) densest nodes before collapse, from low spin $s$ material ( $s$-rich gas particles within these clumps form small disks around the stellar centers; see lower panel). The figure also indicates that by $z_{\text {coll }}$ (middle panel) most spheroid-to-be particles are within the halo and roughly half of them have turned into stars, while part of the disk predecessors are still being assembled as diffuse gas into a gaseous disk, where disk stars will eventually form. Indeed, the

\footnotetext{
6 The differences between thin and thick disk, or those between bulge and stellar halo, are not addressed in this Letter.
} 


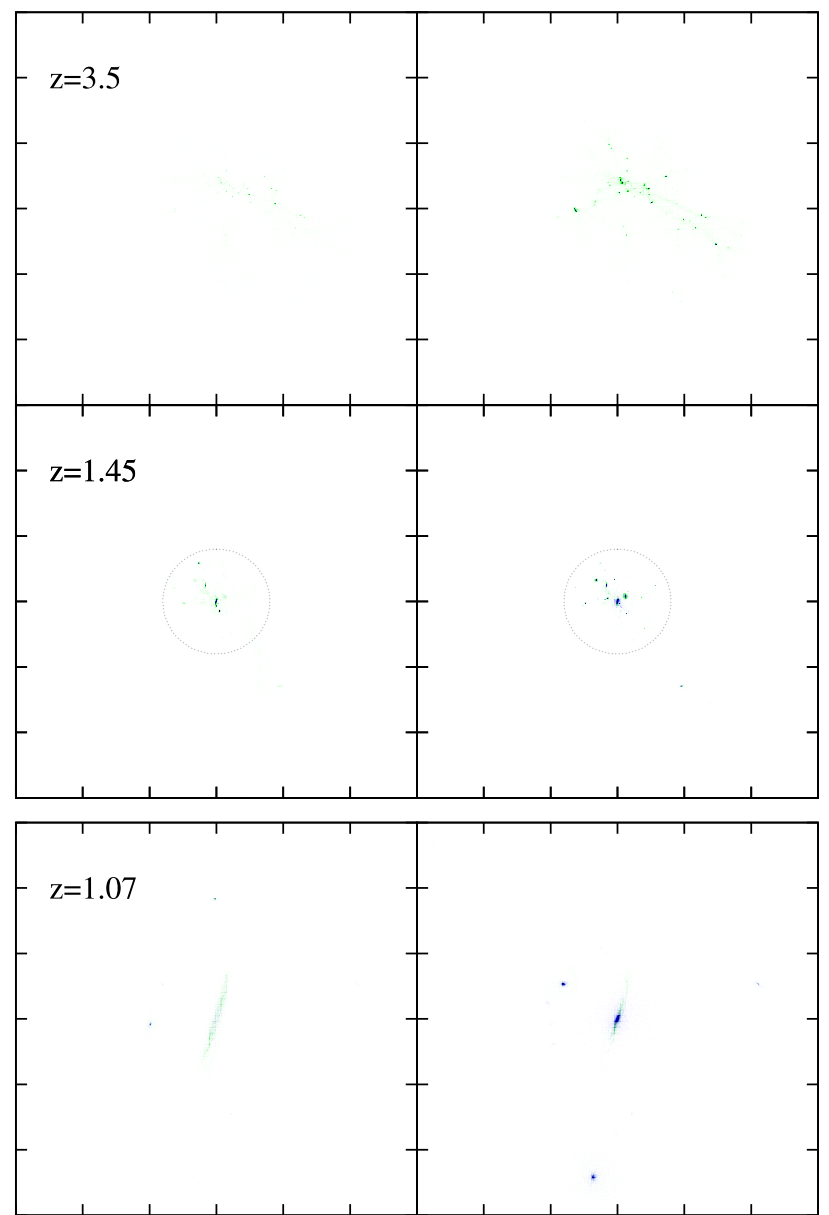

Figure 1. For the HD-5004A galaxy, a projection of the positions of its stellar particles identified at $z=0$ when traced back to $z_{\text {high }}=3.5,1.45$, and 1.07. Left: (thin) disk-to-be particles. Right: spheroid-to-be particles. Green (blue): gaseous (stellar) particles at the respective $z_{\text {high }} s$. From top to bottom the box sides are 600,600 , and $60 \mathrm{kpc}$, respectively. Circles stand for the projection of the virial sphere.

age distributions of different simulated disk galaxies can be found in Doménech-Moral et al. (2012), where this age segregation among the different components is confirmed (see their Figure 10). Concerning baryon mass assembly of the spheroid population, it mostly occurs at $z>z_{\text {coll }}$ through collapse-induced merging, that is, along the so-called fast phase of galaxy evolution, as Obreja et al. (2013) have found. ${ }^{7}$ In this case, as we will see in more detail below, it is the proximity to the patch center that segregates those particles that will be involved in the collapse and suffer from severe $j$ losses from those that will not.

In the lower panel we plot the progenitors at $z=1.07$, when the system has decoupled from expansion and filaments at the virial scale are significantly weaker than at earlier times. At this point, when the proto-disk is settled, gravitational torques appear at the disk scale that tend to redistribute $j$, and to gradually align gaseous particles (either diffuse or in satellites) with the main disk (Danovich et al. 2014) as they dissipate energy due to their $j$ precession. Collisionless mass elements cannot dissipate their energy, therefore they are not prone to being aligned. The important point here is that while well

\footnotetext{
7 These authors found that classical bulges have also a kinematical disky stellar component formed after collapse.
}

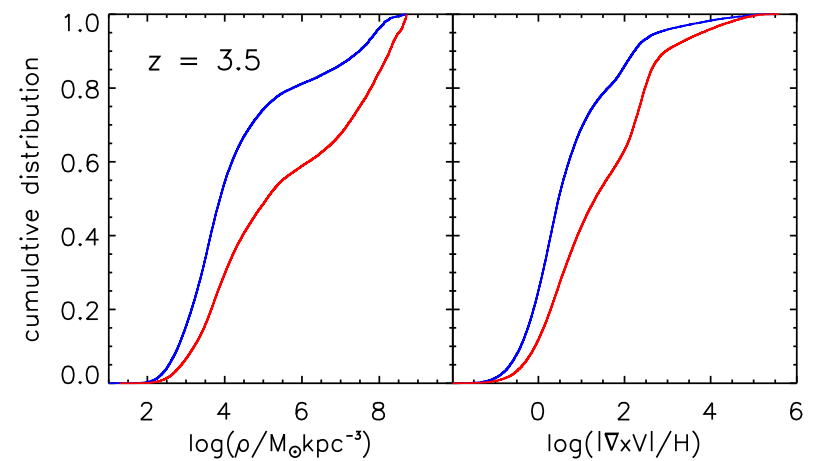

Figure 2. Cumulative PDFs for $\log \left(\rho_{i}\right)$ and $\log \left(w_{i}\right)$ for the HD-5004A galaxy at $z_{\text {high }}=3.5$. Red (blue) lines stand for gaseous spheroid (disk) precursors.

aligned material (either originally or as a consequence of gravitational torques) is incorporated in the disk, the rest of the material is not. An example of such behavior shows up in the lower panel, where we see that a disk originally aligned with the main progenitor is almost entirely incorporated into the thin disk (right panel); however, for two disks that are not parallel to the main at $z=1.07$ only a fraction or their gaseous component goes to the thin disk, while the rest goes to thick disk (not shown) or to the spheroid (right panel). In these cases, the $\mathrm{CW}$ evolution has provided different initial conditions for the torques to act upon.

The visual differences in Figure 1 have been quantified by means of a V-Web-inspired method (Hoffman et al. 2012). For a given simulated galaxy and different $z_{\text {high }} s$, the SPH density $\rho_{i}$ and vorticity $w_{i}$ (measuring the spin of resolution scale whirls) of each gaseous particle, and the distances $r_{i}$ and angular momenta $j_{i}$, relative to the system c.o.m, for gaseous and stellar particles at the $z_{\text {high }} s$ have been calculated, as well as their respective probability distribution functions (PDFs). The results confirm the visual impression that the spheroid-tobe gas particles are the first to be involved in shell-crossing events.

To illustrate these results, in Figure 2 we plot the $\log \left(\rho_{i}\right)$ and $\log \left(w_{i}\right)$ cumulative PDFs at $z_{\text {high }}=3.5$ for the same galaxy as in Figure 1. The PDFs show that, at given times, the spheroidto-be gas particles have the highest densities and vorticities, maping out the $\mathrm{CW}$ element locations. In Figure 3, the $r_{i}$ distributions (first column) confirm that the spheroid-to-be particles (in red) are, at any $z$, closer to the system c.o.m. than the disk progenitors (blue), having therefore a lower probability of being $j$-rich (i.e., lever effect, second column). This is true also at high $z s$ as the first row, giving the corresponding cumulative PDFs of $r_{i}$ and $j_{i}$ for gaseous precursors, illustrates. The relative differences in the $r_{i}$ PDFs are particularly important at the inner region. A K-S test confirms that the red and blue distributions differ at a confidence level higher than $99.8 \%$. We also see in the third row panels that, remarkably, the spheroid-to-be particles are involved in the system collapse by $z \sim 1.5$, so that most of the spheroid-to-be gas particles are within the virial radius $(80 \mathrm{kpc})$ at $z<1.5$, having suffered from severe $j_{i}$ losses at collapse (middle and bottom panels).

By contrast, proto-disk particles are farther away, and therefore not significantly affected by collapse. Many protodisk particles enter the halo as $j$-rich gas particles later on, where they fall to the central regions as they lose $j$ in a more 

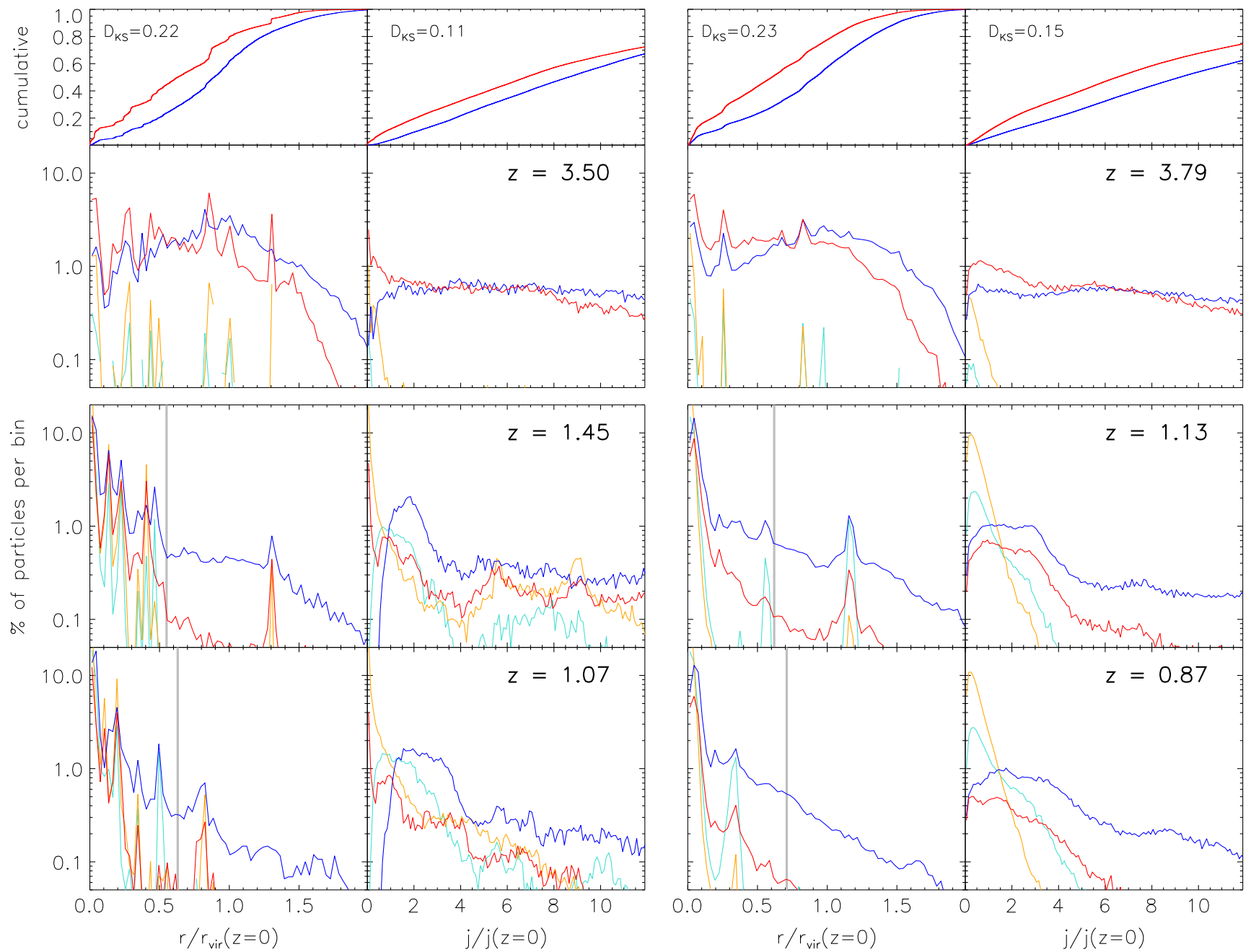

Figure 3. PDFs for $r_{i}$ and $j_{i}$ measured on HD-5004A and g1536- $L^{*}$ galaxies-to-be particles at different $z$ high values, with cumulative ones at the highest $z s$ in the first row. Red (blue) lines stand for gaseous spheroid (disk) precursors, while orange (cyan) lines refer to spheroid (disk) precursors particles that are already stellar at the respective $z_{\text {high }} s$. The vertical normalization has been separately taken for the two kinds of precusors, including in any case both the gas and the stars. Distance units are the virial radii at $z=0$ (138 and $167 \mathrm{kpc}$, respectively), while $j_{i}$ is given in units of the total baryonic (spheroid + disk) specific angular momenta at $z=0$. The thick gray lines correspond to the virial radii at these $z s$.

gradual manner (narrow low $j_{i}$ component at the left). The $j$ content of the gas of the disk component is increased as time goes by, because delayed particles have a higher $j_{i}$ at larger distances (lever effect; see Section 1).

On the right hand side of Figure 3 we show a similar plot for a galaxy in the GASOLINE set. The results are similar to those of the P-DEVA set concerning the $r_{i}$ and $j_{i}$ distribution patterns and their evolution for the two components. ${ }^{8}$ The similarity in the patterns of $j$ evolution are even clearer from Figure 4, where we present the total $j(z) \equiv|\vec{j}(z)|$ for the disk-to-be $\left(j_{\text {disk }}(z)\right.$; blue and cyan) and for the spheroid-to-be $\left(j_{\text {sph }}(z)\right.$; red and magenta) components. We see that $j_{\text {sph }}(z)$ is lower than $j_{\text {disk }}(z)$ for any $z$. We also see that after a gain period, there are severe $j_{\text {sph }}$ losses beginning at $z_{\text {coll }} \sim 1.5$ (1.0) i.e., collapse for HD

5004A (g1536- $\left.L^{*}\right)$, repectively, affecting the gas (in red) and later on the stars (in magenta). On the contrary, $j_{\text {disk,gas }}(z)$ is roughly constant $\left(\mathrm{g} 1536-L^{*}\right)$ or increases after collapse all the

\footnotetext{
8 Some differences exist, however, likely due to the blast wave energy injected in the configuration in the GASOLINE case, preventing high gas densities to develop.
}

way to $z=0$ (HD-5004A), while $j_{\text {disk,star }}(z)$ slightly decreases or keeps constant, showing differences with the gaseous component at $z<0.2$.

\section{SUMMARY AND DISCUSSION}

In summary, the baryons forming current spheroid stellar populations come from gas particles that differ from the protodisk particles. First, prior to collapse, the spheroid progenitors have a higher probability of (1) sampling the shell-crossing regions, thereby forming stars from earlier on, and (2) being closer to the system c.o.m. with a lower $j$ content. Second, because of this proximity, they are involved in the collapse event and lose most of their $j$. Third, once the main disk is settled, spheroid progenitors at the circumgalactic region have not succeeded at aligning their $j$ with it, either because they are more likely to be stars rather than gas, or because of their orbital configuration.

The results, obtained with two codes that are different in their design and subgrid modeling (see Section 2), strongly suggest that, indeed, the different properties of stellar populations in the spheroid and disk express information 


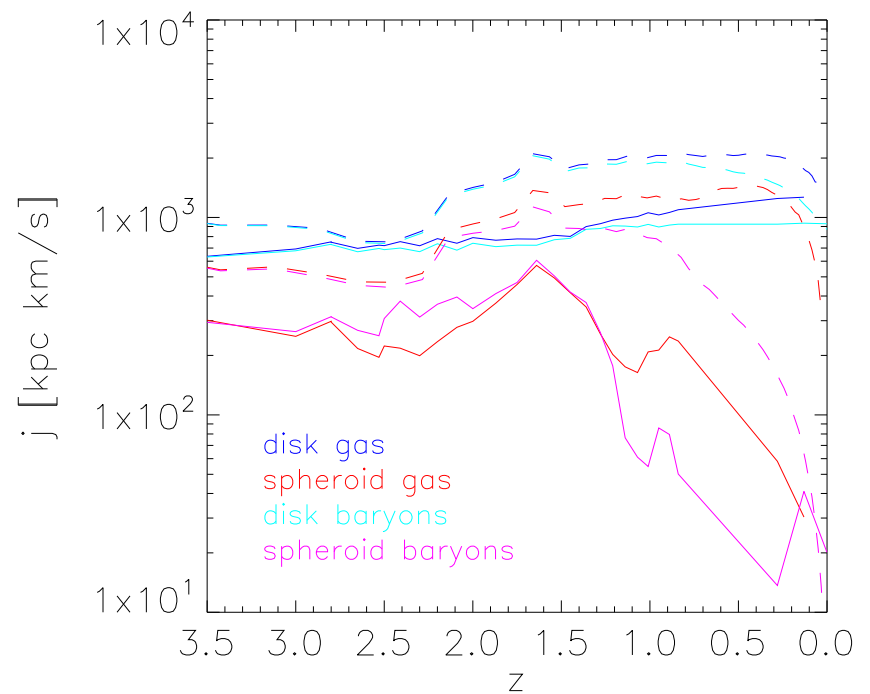

Figure 4. The total specific angular momentum content of HD-5004A (full lines) and g1536- $L^{*}$ (dashed lines) galaxies-to-be particles as a function of $z$. Red (blue) lines stand for gaseous spheroid (disk) precursors, while magenta (cyan) lines refer to spheroid (disk) precursors particles that are either stellar or gaseous at the respective $z s$.

imprinted at high $z$ (prior to collapse) while they displayed a $\mathrm{CW}$ configuration, and that this information comes from a fundamental level of physical description, as encapsulated within the Zel'dovich approximation and the adhesion model; see Section 1.

Gas $j$ content changes take place at collapse, and later on within the circumgalactic region (e.g., disk-induced alignments, among many other processes), changes that can remove the imprints of CW dynamics left at these smaller scales. In this respect, our results show that the spheroid and disk stellar populations of local galaxies (i.e., at disk scale) keep memory of the CW dynamics at high $z$. This strongly suggests that, in the long run, the ordering imprinted on the cosmic inflow (that provides with segregated initial conditions for lower $z$ processes) overcomes the complex astrophysical processes occurring in the circumgalactic environment of forming disks.

We thank M. Doménech for allowing us to use results of simulations. We thankfully acknowledge the computer resources provided by BSC/RES (Spain). The Centro de Computación Cientifíca (UAM, Spain) has also provided computing facilities. This work was partially supported by the MICINN and MINECO (Spain) through the grants AYA200912792-C03-02, AYA2009-12792-C03-03, and AYA201231101 from the PNAyA, as well as by the "Supercomputación y e-Ciencia" Consolider-Ingenio CSD2007-0050 project. A.O. was financially supported through a FPI contract from AYA2009-12792-C03-03 and C.B.B. through a contract from AYA2012-31101.

\section{REFERENCES}

Agertz, O., Teyssier, R., \& Moore, B. 2011, MNRAS, 410, 1391

Binney, J. 1977, ApJ, 215, 492

Birnboim, Y., \& Dekel, A. 2003, MNRAS, 345, 349

Bond, J. R., Kofman, L., \& Pogosyan, D. 1996, Natur, 380, 603

Brook, C. B., Stinson, G., Gibson, B. K., Wadsley, J., \& Quinn, T. 2012a, MNRAS, 424, 1275

Brook, C. B., Stinson, G. S., Gibson, B. K., et al. 2012b, MNRAS, 426, 690 Brooks, A. M., Governato, F., Quinn, T., Brook, C. B., \& Wadsley, J. 2009, ApJ, 694, 396

Cautun, M., van de Weygaert, R., Jones, B. J. T., \& Frenk, C. S. 2014, MNRAS, 441, 2923

Codis, S., Pichon, C., Devriendt, J., et al. 2012, MNRAS, 427, 3320

Danovich, M., Dekel, A., Hahn, O., Ceverino, D., \& Primack, J. 2014, MNRAS, submitted (arXiv:1407.7129)

Danovich, M., Dekel, A., Hahn, O., \& Teyssier, R. 2012, MNRAS, 422, 1732

Dhillon, I., Guan, Y., \& Kulis, B. 2004, A unified view of kernel k-means, spectral clustering and graph cuts Technical Report TR-04-25, Univ. Texas at Austin, Department of Computer Sciences

Doménech-Moral, M., Martínez-Serrano, F. J., Domínguez-Tenreiro, R., \& Serna, A. 2012, MNRAS, 421, 2510

Domínguez-Tenreiro, R., Oñorbe, J., Martínez-Serrano, F., \& Serna, A. 2011, MNRAS, 413, 3022

Domínguez-Tenreiro, R., Obreja, A., Granato, G. L., et al. 2014, MNRAS, 439, 3868

Doroshkevich, A. G. 1970, Ap, 6, 320

Dubois, Y., Pichon, C., Welker, C., Le Borgne, D., et al. 2014, MNRAS, 444, 1453

Gilmore, G., Wyse, R. F. G., \& Kuijken, K. 1989, ARA\&A, 27, 555

Gurbatov, S. N., Saichev, A. I., \& Shandarin, S. F. 1989, MNRAS, 236, 385

Gurbatov, S. N., Saichev, A. I., \& Shandarin, S. F. 2012, PhyU, 55, 223

Hoffman, Y., Metuki, O., Yepes, G., et al. 2012, MNRAS, 425, 2049

Ivezić, Ž., Beers, T. C., \& Jurić, M. 2012, ARA\&A, 50, 251

Karatzoglou, A., Smola, A., \& Hornik, K. 2004, J. Stat. Softw., 9, 1

Kereš, D., Katz, N., Fardal, M., Davé, R., \& Weinberg, D. H. 2009, MNRAS, 395,160

Kereš, D., Katz, N., Weinberg, D. H., \& Davé, R. 2005, MNRAS, 363, 2

Kimm, T., Devriendt, J., Slyz, A., et al. 2011, MNRAS, submitted (arXiv:1106.0538)

Kofman, L., Pogosian, D., \& Shandarin, S. 1990, MNRAS, 242, 200

Martínez-Serrano, F. J., Serna, A., Domínguez-Tenreiro, R., \& Mollá, M. 2008, MNRAS, 388,39

Mould, J. R. 1982, ARA\&A, 20, 91

Obreja, A., Brook, C. B., Stinson, G., et al. 2014, MNRAS, 442, 1794

Obreja, A., Domínguez-Tenreiro, R., Brook, C., et al. 2013, ApJ, 763, 26

Ocvirk, P., Pichon, C., \& Teyssier, R. 2008, MNRAS, 390, 1326

Peebles, P. J. E. 1969, ApJ, 155, 393

Pichon, C., Pogosyan, D., Kimm, T., et al. 2011, MNRAS, 418, 2493

Sales, L. V., Navarro, J. F., Theuns, T., et al. 2012, MNRAS, 423, 1544

Schölkopf, B., Smola, A., \& Müller, K.-R. 1998, Neural Comput., 10, 1299

Serna, A., Domínguez-Tenreiro, R., \& Sáiz, A. 2003, ApJ, 597, 878

Stewart, K. R., Brooks, A. M., Bullock, J. S., et al. 2013, ApJ, 769, 74

Stinson, G., Brook, C., Macciò, A. V., et al. 2013, MNRAS, 428, 129

Stinson, G., Seth, A., Katz, N., et al. 2006, MNRAS, 373, 1074

Talbot, R. J., Jr., \& Arnett, W. D. 1973, ApJ, 186, 51

Tillson, H., Devriendt, J., Slyz, A., Miller, L., \& Pichon, C. 2012, MNRAS, submitted (arXiv:1211.3124)

van de Voort, F., Schaye, J., Booth, C. M., Haas, M. R., \& Dalla Vecchia, C. 2011, MNRAS, 414, 2458

Wadsley, J. W., Stadel, J., \& Quinn, T. 2004, NewA, 9, 137

Welker, C., Devriendt, J., Dubois, Y., Pichon, C., \& Peirani, S. 2014, MNRAS, 445, L46

White, S. D. M. 1984, ApJ, 286, 38

Zel'dovich, Y. B. 1970, A\&A, 5, 84 